Thorax (1973), 28, 177.

\title{
Experience with Devices implanted fixed-rate pacemakers
}

\author{
HAROLD SIDDONS and GEOFFREY DAVIES
}

\section{St. George's Hospital, London S.W.1}

The fate of 183 implants of Devices TF 2970 fixed-rate pacemakers is analysed. The incidence of clinical complications such as sepsis or skin necrosis, displacement of the electrode, and exit block, and the causes of the 19 deaths are discussed.

In 28 of the 183 pacemakers pacing stopped for technical reasons, which are described; the commonest cause of failure was the batteries.

The incidence of pacemaker failure has been estimated statistically from a much larger series of 558 implants.

Twelve years' experience shows that the life of a pacemaker in clinical conditions cannot be predicted from the maker's information. It is necessary to wait until a considerable number have run their life's span as implants. Modification of design of new models comes on the market so frequently that by the time clinical experience has accumulated on one model it is often superseded. Devices Ltd. introduced their fixed-rate model TF 2970 in November 1968. By the end of March 1970 we had implanted 184 of these using endocardial electrodes. We know the fate of all except for one implanted in a patient who was transferred to another hospital's care. The model 2970 is still in production, though it has undergone some minor modifications.

This paper reports the fate of these pacemakers after implantation. It is followed by a paper (Davies and Siddons, 1973) on the prediction of the life of a pacemaker once it has been implanted, reporting in detail the method which we have developed and are now using at St. George's Hospital.

Table I classifies the various fates of 183 of these implants, excluding the one transferred to another hospital for after-care.

Sepsis or necrosis of the skin over the pacemaker required removal of the pacemaker in 14 patients. The change of pacemaker was required within the first three months in only four of these, and in a considerable proportion the process was clearly mechanical rather than septic. The one septicaemia followed a previous implantation for which the electrode wire had ulcerated
T A B L E I

FATE OF 183 PACEMAKERS

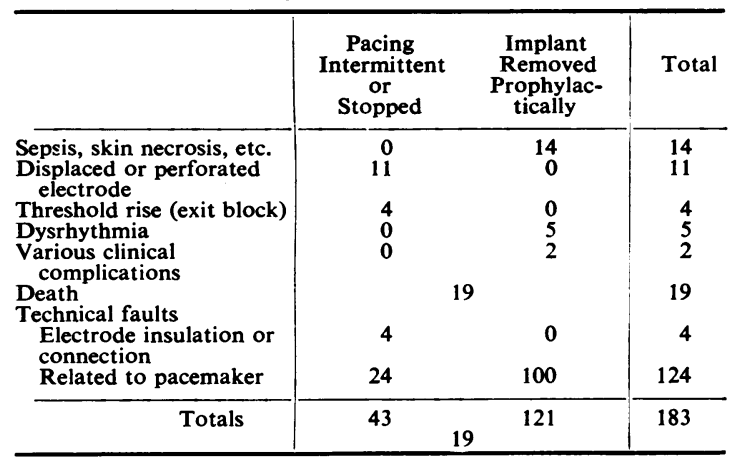

and allowed bacterial access to the venous system. A new contralateral system was put in but the septicaemia was not at first controlled ; the endocardial system was therefore removed and pacing was successfully continued with myocardial electrodes.

Perforation or displacement occurred in 11 patients. The intracardiac electrode occasionally penetrates through the right ventricular wall. This usually results in cessation of pacing, but in no case was there any other significant clinical effect. Perforation is usually clinically indistinguishable from electrode displacement. Five of the 11 displacements occurred in the first five days, the remainder within the next six weeks. Out of our last 350 electrode introductions repositioning has been required on 36 occasions. 
In four patients failure to pace was due to 'exit block' (threshold rise). It is possible that some of these failures may have resulted from late displacement of the electrode tip. Manipulation or change of electrode resulted in satisfactory pacing in all.

In five patients dysrhythmias competing with the pacemaker caused anxiety. In all these the pacemaker was at least temporarily brought out so that demand and various rates of pacing could be tried. Control was not achieved in one of them, the patient dying nine days later. In one, defibrillation was required for ventricular fibrillation and the pacemaker was damaged by the repeated shocks. This patient survives.

The two patients in Table I under the heading 'various clinical complications' were (1) a child in whom growth necessitated lengthening the electrode wire; at operation the opportunity to provide a new pacemaker was taken; and (2) a patient with dizzy spells which it was thought might have been due to pacemaker malfunction, though this was subsequently disproved.

Nineteen patients died. Fourteen of these deaths were classified as unrelated to pacing though in one of them the pacing procedure may possibly have precipitated the fatal cerebrovascular accident 11 days after implantation. One patient suffered a series of bouts of dysrhythmia both before and after pacing and died suddenly 16 days after a change of pacemaker. Two later deaths followed shortly after an incident of collapse. Although these patients did not reach hospital for diagnoses to be made it is possible that the collapse and subsequent death resulted from failure of pacing. The other two patients died suddenly.

The above various clinical reasons for cessation of pacing account for 55 of the 183 failures, leaving 128 , in 28 of which pacing stopped and 100 pacemakers were removed prophylactically.

Table II shows the technical causes of failure to pace in 28 cases. It is possible that the failure in three cases might have resulted from surgical damage on dissecting free embedded wires at the

T A B L E I I

TECHNICAL CAUSES OF FAILURE TO PACE

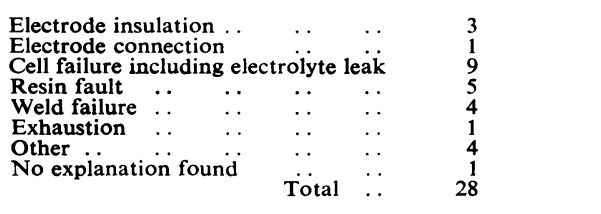

change of pacemaker. It is remarkable that in only one patient was cessation of pacing due to exhaustion of all four cells of the battery. Nine of the pacemaker failures resulted from the electrolyte $\overrightarrow{\widetilde{D}}$ of the battery either forcing the battery cap to 2 separate or leaking and damaging other parts of $\$$ the pacemaker. This inherent defect of the $\overrightarrow{0}$ currently used cells has led many manufacturers to search for other power sources which can be $\vec{\omega}$ hermetically sealed.

The oscilloscopic method of predicting pace- $\vec{x}$ maker failure described by Davies and Siddons $\mathbb{N}_{\infty}$ (1973) has not only proved successful in forecast- ? ing true battery depletion when all four of the $\vec{\forall}$ Mallory cells are reaching exhaustion, but has also revealed when failure of one of the four cells has occurred.

Unfortunately we have been unable to predict this premature failure of a single cell which occurs $\frac{\widehat{O}}{\partial}$ over a few days and has been the commonest $\overrightarrow{0}$ pacemaker fault in this series. When the test has shown failure of one cell it has been taken as $\omega$ an indication to change the unit. The design of the pacemaker has been altered in order to circumvent this particular fault though it is as yet too early to record how successful the modified pacemaker will be.

Our overall experience of this defect has been in $32 \overrightarrow{\overrightarrow{0}}$ units; 26 of these had makers' numbers between 3 A 220 and A 999, representing $21 \%$ of the 123 pacemakers supplied to us within this number range. The battery cell type for all these was a particular model in Mallory's range of certified medical grade cells and $\bar{\partial}$ this model is no longer used in Devices pacemakers. In spite of a change to a cell of different construction this defect has so far occurred in 16 of 400 pacemakers 3 with other cell types. Premature cell failure has occurred also in Medtronic pacemakers implanted in $\mathrm{O}$ this hospital, and a premature failure rate of $12 \%$ by 14 months in a series of Medtronic pacemakers has 윽 been reported in Paris (Welti et al., 1972).

The Figure shows the time incidence of the various clinical indications for removal as com- $N$ pared with the deaths and technical indications. N The criteria for prophylactic removal had not been 0 defined early in the series and a number were $\omega$ removed at an unnecessarily early stage. Moreover, when a pacemaker had been implanted for 180 months and the patient wished to travel abroad or $\mathbb{D}$ take a holiday which would prevent the usual ${ }^{+}$ supervision, the pacemaker was changed.

The data in the Figure show the incidence of $\frac{\circ}{\circ}$ technical failure in the pacemaker system. In the $\frac{\rho}{\odot}$ first six months there was only one breakdown due $\stackrel{\mathbb{2}}{2}$ to failure to make a good sound connection between the electrode wire and the unit. From $6 \%$ 


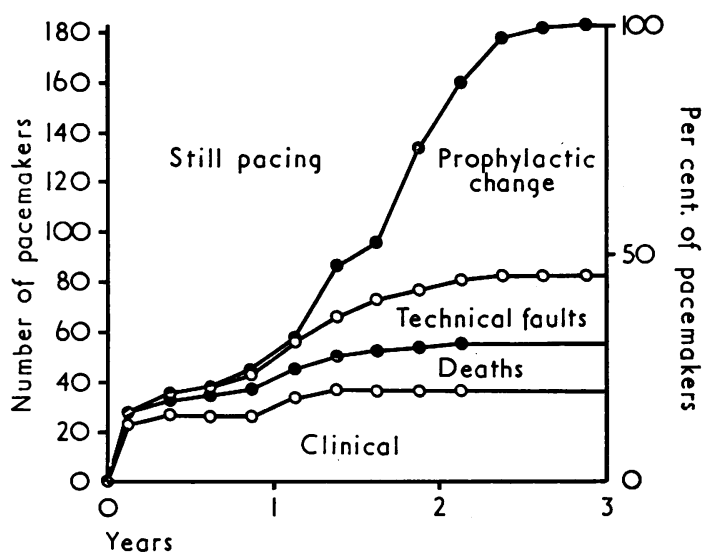

FIGURE. Fate of 183 implants.

months up to 27 months the incidence of failure is remarkably constant.

Statistical analysis of this series augmented by the pacemakers of the same model which we implanted between 1 April 1970 and 30 August 1972 (total 558) shows that the risk of pacemaker failure occurring in any three-month period is as follows:

Up to 6 months-one failure

6 to 15 months $-0.7 \%$ (95\% confidence levels $0.51 \%$ to $0.91 \%$ )

15 to 27 months $-4.6 \%$ (95\% confidence levels $3.84 \%$ to $5.28 \%$ )

27 to 30 months $-9 \cdot 1 \%$.

Devices Ltd. introduced their Demand models in 1969, the year after the fixed-rate pacemakers, but the model has on several occasions since been changed, and our experience with any of the earlier models is too limited for valuable analysis. The current model has not been available long enough for the clinical results to be appraised. Experience so far suggests that its reliability and life are not greatly different from those of the fixed-rate model.

\section{CONCLUSIONS}

Displacement of the electrode tip within the first few days or up to six weeks after implantation is the common early cause of failure to pace. Faults in the pacemaker itself begin to appear after six months. The commonest of them is failure of a single battery cell, as opposed to the expected depletion of all four cells. This can usually be determined, before pacing ceases, by the oscilloscopic test described by Davies and Siddons. It is thought that this defect, which was common in pacemakers produced over a particular period, has been almost eliminated by alteration in design.

So far only one unit has been allowed to reach battery depletion. Of the pacemakers removed for technical reasons either prophylactically or because of the cessation of pacing, half were removed by 21 months. This figure represents the situation before the oscilloscope test was being applied ; and laboratory testing shows that many of these units had sufficient battery power to last considerably longer. It appears that, if useful life depended only on normal battery depletion, they could have been allowed to stay implanted much longer, perhaps to a mean period of 36 months. We are confident that, in each individual, the oscilloscopic test can predict this span of life, and should allow in the future $90 \%$ of this life to be used.

\section{REFERENCES}

Davies, G., and Siddons, H. (1973). Prediction of battery depletion in implanted pacemakers. Thorax, 28, 180.

Welti, J., Bonnet, M., Stoltz, J. P., Kevorkian, M., Pioger, G., and Fontaine, G. (1972). Usure et pannes des pacemakers. Arch. Mal. Coeur, 65, 1277. 